\title{
Recent Advances in Kernel Machines
}

\author{
Stéphane Canu \\ LITIS - INSA Rouen - France \\ stephane.canu@insa-rouen.fr \\ http://asi.insa-rouen.fr/enseignants/ scanu/
}

\begin{abstract}
This talk will review recent advances in the kernel methods focusing on support vector machines (SVM) for pattern recognition. Topics discussed include the kernel design issue through the multi kernel approach and the optimization issue with emphasis on scalability and non convex cost functions.
\end{abstract}

